\title{
Dynamique en flexion de tubes parcourus à grandes vitesses
}

\author{
Quentin LamberT ${ }^{1,2, a}$, André Langlet ${ }^{1}$, Jérôme Renard ${ }^{1}$ Et Nicolas Eches ${ }^{3}$ \\ 1 Institut PRISME, UPRES 4229/Équipe Risques Explosions Structures, Université d'Orléans, 63 avenue de Lattre de Tassigny, \\ 18020 Bourges Cedex, France \\ 2 C.T.A. International, 8 route de Guerry, 18023 Bourges Cedex, France \\ 3 NEXTER Munition, 7 route de Guerry, 18023 Bourges Cedex, France
}

Reçu le 30 septembre 2008, accepté le 18 décembre 2008

\begin{abstract}
Résumé - Ce travail porte sur la réponse temporelle d'une poutre à section et inertie variable, sollicitée par un chargement mobile. Dans un premier temps le chargement est apporté par une masse contrainte de se déplacer sur la poutre sans pouvoir s'en séparer. Dans un deuxième temps, le chargement est apporté par un oscillateur en mouvement sur la poutre. Cette modélisation sera confrontée à d'autres codes de calculs tels que LS-Dyna et SIMBAD. L'application concerne la dynamique des tubes d'armes couplée au projectile pendant son parcours balistique dans le tube. L'exploitation des modèles présentés a pour but d'obtenir une approximation des paramètres de sortie du projectile, lesquels, en constituant les conditions initiales du vol, influencent la dispersion à la cible.
\end{abstract}

Mots clés : Poutre de Timoshenko non uniforme / dynamique rapide / charge mobile / interaction masse mobile-poutre / interaction oscillateur mobile-poutre

Abstract - Flexural dynamics of tubes traversed by high speed interval moving loads. In this work, the time-response of a beam, of variable cross-section and inertia, submitted to a moving load, is studied. Firstly, the response of the beam to a moving mass is sought; secondly, the response of the beam to a moving oscillator. The application of this work concerns the dynamics of a gun barrel traversed at high velocities by the projectile. The results of the models enable to approximate the exit conditions which provide the initial flight conditions that influence the impact dispersion at the target.

Key words: Non uniform Timoshenko beam / fast dynamics / moving load / moving mass-beam interaction / moving oscillator-beam interaction

\section{Introduction}

Déterminer la réponse des structures parcourues par des chargements variables est un problème d'une grande importance industrielle. Les nombreuses applications se rencontrent dans les domaines du génie civil couplé à celui des transports (ponts traversés par des véhicules, trains à grandes vitesses, pistes de porte-avions, pistes flottantes, mouvements de la charge sur le câble d'une grue, etc.), de la conception des machines d'usinage, mais aussi de la dynamique des tubes d'armes, ou encore de la réponse des structures parcourues par des ondes de choc. C'est pour cette raison que le problème des chargements mobiles est à l'origine de nombreuses études théoriques. Comme le rappelle Lee [1], depuis les premiers travaux de Stokes en 1849 , de très nombreuses études ont été publiées. Pesterev et al. [2] ont présenté récemment une synthèse. La structure type étudiée est une poutre : généralement suivant

\footnotetext{
a Auteur pour correspondance :

Quentin.Lambert@bourges.univ-orleans.fr
}

la théorie de Euler-Bernoulli, parfois suivant la théorie de Timoshenko. On distingue trois approches pour exprimer l'excitation sur la poutre : (i) le chargement est constitué par une force d'intensité constante se déplaçant à une vitesse $V(t)$ fonction du temps; (ii) le chargement est constitué par une masse se déplaçant sur la poutre; (iii) le chargement est constitué par un oscillateur en mouvement.

Le premier cas (i) ne comporte pas de couplage : la solution est issue de la résolution des équations de la poutre excitée par une force $F_{0} \delta\left(x-x_{\mathrm{c}}(t)\right)(\delta(\cdot)$ représente la distribution de Dirac et $x_{\mathrm{c}}(t)$ l'abscisse du point d'application de la force). Bien que conceptuellement le plus simple, le cas d'une excitation par une force constante est intéressant théoriquement car il permet d'obtenir une solution analytique stationnaire (Steele [3], Fryba [4], Renard et al. [5]). Or, sous certaines conditions, la solution stationnaire est la solution vers laquelle tend la réponse observée : en effet, si une structure suffisamment étendue est traversée à vitesse constante par une 


\section{Nomenclature des principales notations}

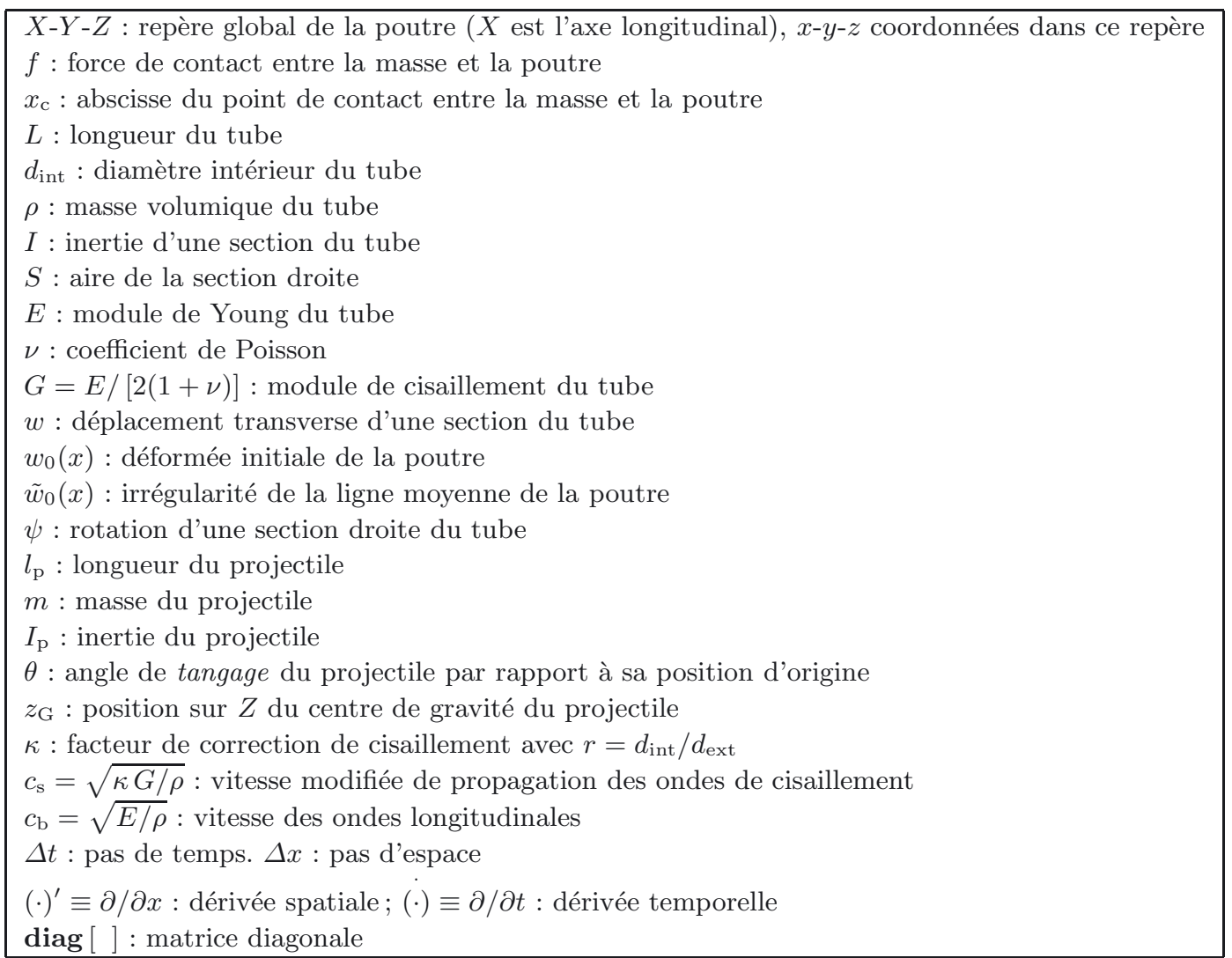

force, pendant une durée assez longue, les ondes élastiques s'organisent de manière régulière dans l'espace et dans le temps pour apparaître progressivement comme figées à l'observateur lié à la force en mouvement. Dès lors, la recherche des solutions stationnaires est indissociable de l'étude des chargements mobiles.

Lorsque le chargement est constitué par une masse $m$ en mouvement (ii), la force contient des termes d'inertie traduisant l'interaction entre la structure et la masse. Si on considère que la masse est contrainte de se déplacer sur la poutre sans pouvoir s'en séparer (l'éventualité d'une séparation est traitée par Lee [1]), la force de contact $f$ comprend le poids $m g$ et les termes d'inertie : force centrifuge, force de Coriolis, termes dépendant du temps par la loi de vitesse :

$$
\begin{aligned}
f & =m\left[g-d^{2} w_{t} / d t^{2}\right] \\
& =m\left[g-\ddot{w}_{t}-2 V \dot{w}_{t}^{\prime}-V^{2} w_{t}^{\prime \prime}-\dot{V} w_{t}^{\prime}\right]_{x=x_{\mathrm{c}}}
\end{aligned}
$$

où $w_{t}$ est le déplacement transverse de la poutre. Avec une déformée initiale de la poutre telle que : $w_{t}(x, t)=$ $w(x, t)+w_{0}(x)$, on $\mathrm{a}:$

$$
f=m\left[g-V^{2} w_{0}^{\prime \prime}-\dot{V} w_{0}^{\prime}\right]-m\left[\ddot{w}+2 V \dot{w}^{\prime}+V^{2} w^{\prime \prime}+\dot{V} w^{\prime}\right]
$$

La solution de ce problème est intéressante car elle permet de se rendre compte de l'importance relative des effets des paramètres suivants : loi de vitesse $V(t)$, flexibilité de la structure, rapport entre la masse mobile et celle de la structure, déformée initiale représentée par les termes en $w_{0}$. La résolution est difficile et a conduit à des approximations. Ainsi, on peut se ramener au cas (i) si tous les termes d'inertie sont négligeables devant le poids $m g$. D'autre part, on peut négliger les termes centrifuge et de Coriolis figurant dans l'accélération si la vitesse $V(t)$ et son accélération $\dot{V}(t)$ sont suffisamment faibles. Enfin, dans la plupart des travaux publiés, la masse se déplace à vitesse constante. La solution du problème est recherchée par l'intermédiaire de méthodes approchées : méthode des éléments-finis (Rieker et al. [6], Cartmell et al. [7], par exemple), développement en série de modes propres (Olsson [8], Lee [1], Lee [9], Akin et al. [10]), formulation des équations du mouvement sous forme intégro-différentielle à l'aide des fonctions de Green (Ting et al. [11]) puis résolution numérique (différences finies par Ting et al.).

Enfin dans le cas (iii), la masse en mouvement est reliée à la structure par un ressort de raideur $k$ et éventuellement un amortisseur de constante $c$. La force de contact, action de la masse sur le tube s'écrit alors :

$$
\begin{aligned}
f= & \left\{m g+k\left[z(t)-w\left(x_{\mathrm{c}}, t\right)\right]+c\left[\dot{z}(t)-\dot{w}\left(x_{\mathrm{c}}, t\right)\right\}\right. \\
& \times \delta\left(x-x_{\mathrm{c}}\right)
\end{aligned}
$$

avec l'équation supplémentaire :

$$
m \ddot{z}=-k\left[z(t)-w\left(x_{\mathrm{c}}, t\right)\right]-c\left[\dot{z}(t)-\dot{w}\left(x_{\mathrm{c}}, t\right)\right]
$$


Le modèle de la poutre parcourue par un oscillateur prend en compte la liaison de la poutre avec la masse en mouvement. Les conditions d'équivalence des trois modèles sont discutées par Pesterev et al. [12]. En particulier, les influences des conditions initiales (déplacements et vitesses initiales) et des conditions limites sur les conditions d'équivalence sont clairement présentées.

Dans cet article, on s'intéresse aux mouvements de flexion d'une poutre à section et inertie variables sous l'influence d'un chargement mobile. L'application concerne la dynamique des tubes d'armes couplée au parcours balistique du projectile dans le tube. Il sera d'abord présenté une discrétisation par éléments-finis permettant d'une part de trouver les modes et fréquences propres et d'autre part de calculer la réponse de la poutre à la masse en mouvement. Dans un second temps, le développement en modes propres permet le calcul de la force d'interaction par superposition modale. Enfin, on présente le modèle de la poutre parcourue par un oscillateur. L'exploitation des modèles présentés a pour but d'obtenir une approximation des paramètres de sortie du projectile, lesquels, en constituant les conditions initiales du vol, influencent la dispersion à la cible.

\section{Modes et fréquences propres de la poutre de Timoshenko tronconique}

On adopte ici l'élément présenté par To [13] et Hou et al. [14] pour discrétiser la poutre. Soit $L_{\mathrm{e}}$ la longueur de l'élément, $\xi=x_{\mathrm{e}} / L_{\mathrm{e}}$ l'abscisse longitudinale réduite dans le repère local de l'élément « $\mathrm{e} »$, dont les nœuds gauche et droit sont notés : $i », \ll j »$, d'abscisses respectives $x_{i}$ et $x_{j}$ sur la poutre. Les degrés de liberté sont : $w_{i}$, $\psi_{i}=\left.\frac{\partial w}{\partial x}\right|_{i}+s_{i}, s_{i}$, où : $w_{i}$ représente le déplacement transverse, $\psi_{i}$ représente la rotation de la section droite $i$ de la poutre fléchie, $s_{i}$ représente l'angle de gauchissement dû au cisaillement. Le vecteur des déplacements nodaux

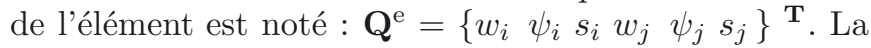
poutre est constituée d'un matériau élastique, homogène et isotrope. Sa section droite variable est annulaire, avec un diamètre intérieur constant : le diamètre extérieur $d_{\text {ext }}$ varie linéairement le long de la poutre, l'aire de section $S$ et le moment d'inertie $I$ par rapport à $y$ sont donnés par :

$$
\begin{gathered}
d_{\mathrm{ext}}(x)=A_{\mathrm{d}} x+B_{\mathrm{d}} \\
B_{\mathrm{d}}=d_{\mathrm{ext}}(0) \\
S(x)=\frac{\pi}{4}\left(d_{\mathrm{ext}}^{2}-d_{\mathrm{int}}^{2}\right), I(x)=\frac{\pi}{64}\left(d_{\mathrm{ext}}^{4}-d_{\mathrm{int}}^{4}\right)
\end{gathered}
$$

Dans le système de coordonnées locales de l'élément, le diamètre extérieur $d_{\text {ext }}$, l'aire de section $S$ et le moment d'inertie $I$ par rapport à $y$ sont donnés par :

$$
\begin{aligned}
d_{\text {ext }}(\xi) & =A_{\mathrm{d}} \xi+B^{\mathrm{e}} \\
B^{\mathrm{e}} & =d_{\text {ext }}(\xi=0) \text { ou } B^{\mathrm{e}}=d_{\text {ext }}\left(x_{i}\right) \\
S(\xi) & =A_{i}\left(1+\gamma_{1} \xi+\gamma_{2} \xi^{2}\right) \\
I(\xi) & =I_{i}\left(1+\delta_{1} \xi+\delta_{2} \xi^{2}+\delta_{3} \xi^{3}+\delta_{4} \xi^{4}\right)
\end{aligned}
$$

Suivant la formulation présentée par To [13], qui est également reprise par Hou et al. [14], on pose : $w=$ $\sum_{k=1}^{4} a_{k}\left(L_{\mathrm{e}} \xi\right)^{k}=\boldsymbol{\Phi}_{\mathbf{w}} \boldsymbol{a}_{\mathbf{w}}, \psi=\Phi_{\psi} a_{\psi}, s=\boldsymbol{\Phi}_{\mathrm{s}} a_{\mathrm{s}}$, où : $\boldsymbol{a}$ représente un vecteur de coefficients du polynôme de la fonction d'interpolation considérée.

$$
\begin{aligned}
& w=\left[\begin{array}{llllll}
1 & L_{\mathrm{e}} \xi & L_{\mathrm{e}}^{2} \xi^{2} & L_{\mathrm{e}}^{3} \xi^{3} & 0 & 0
\end{array}\right] \mathbf{C}^{-\mathbf{1}} \mathbf{Q}^{\mathbf{e}} \equiv \boldsymbol{N}_{\mathbf{w}} \mathbf{Q}^{\mathbf{e}} \\
& \psi=\left[\begin{array}{lllllll}
0 & 1 & 2 L_{\mathrm{e}} \xi & 3 L_{\mathrm{e}}^{2} \xi^{2} & 1 & L_{\mathrm{e}} \xi
\end{array}\right] \mathbf{C}^{-\mathbf{1}} \mathbf{Q}^{\mathbf{e}} \equiv \boldsymbol{N}_{\psi} \mathbf{Q}^{\mathbf{e}} \\
& s=\left[\begin{array}{lllllll}
0 & 0 & 0 & 0 & 1 & L_{\mathrm{e}} \xi
\end{array}\right] \mathbf{C}^{-\mathbf{1}} \mathbf{Q}^{\mathbf{e}} \equiv \boldsymbol{N}_{\mathbf{s}} \mathbf{Q}^{\mathbf{e}}
\end{aligned}
$$

Les vecteurs $\boldsymbol{N}$ représentent les fonctions d'interpolations, que l'on peut expliciter par les produits : $\boldsymbol{N}=$ $\mathbf{\Phi} \mathbf{C}^{-\mathbf{1}}$, avec la matrice $\mathbf{C}^{-\mathbf{1}}$ :

$$
\mathbf{C}^{-1}=\left[\begin{array}{cccccc}
1 & 0 & 0 & 0 & 0 & 0 \\
0 & 1 & -1 & 0 & 0 & 0 \\
-3 / L_{\mathrm{e}}^{2} & -2 / L_{\mathrm{e}} & 2 / L_{\mathrm{e}}^{2} & -3 / L_{\mathrm{e}} & -1 / L_{\mathrm{e}} & 1 / L_{\mathrm{e}} \\
2 / L_{\mathrm{e}}^{3} & 1 / L_{\mathrm{e}}^{2} & -1 / L_{\mathrm{e}}^{2} & -2 / L_{\mathrm{e}}^{2} & 1 / L_{\mathrm{e}}^{2} & -1 / L_{\mathrm{e}}^{2} \\
0 & 0 & 1 & 0 & 0 & 0 \\
0 & 0 & -1 / L_{\mathrm{e}} & 0 & 0 & 1 / L_{\mathrm{e}}
\end{array}\right]
$$

La matrice de masse de l'élément s'écrit : $\mathbf{m}^{\mathbf{e}}=\mathbf{m}_{\mathbf{f}}+\mathbf{m}_{\mathbf{r}}$, où $\mathbf{m}_{\mathbf{f}}$ représente les termes d'inertie de flexion et $\mathbf{m}_{\mathbf{r}}$ représente les termes d'inertie de rotation. On a :

$$
\begin{aligned}
& \mathbf{m}_{\mathbf{f}}=\int_{0}^{L_{\mathrm{e}}} \rho S(x) \mathbf{C}^{-\mathbf{1} \mathbf{T}} \boldsymbol{\Phi}_{\mathbf{w}}^{\mathbf{T}} \boldsymbol{\Phi}_{\mathbf{w}} \mathbf{C}^{-\mathbf{1}} \mathrm{d} x \\
& \mathbf{m}_{\mathbf{r}}=\int_{0}^{L_{\mathrm{e}}} \rho I(x) \mathbf{C}^{-\mathbf{1} \mathbf{T}} \boldsymbol{\Phi}_{\psi}^{\mathbf{T}} \boldsymbol{\Phi}_{\psi} \mathbf{C}^{-\mathbf{1}} \mathrm{d} x
\end{aligned}
$$

La matrice de rigidité de l'élément s'écrit : $\mathbf{k}^{\mathbf{e}}=\mathbf{k}_{\mathbf{f}}+\mathbf{k}_{\mathbf{s}}$, où $\mathbf{k}_{\mathbf{f}}$ représente la rigidité en flexion et $\mathbf{k}_{\mathbf{s}}$ représente celle en cisaillement. On a :

$$
\begin{aligned}
& \mathbf{k}_{\mathbf{f}}=\int_{0}^{L_{\mathrm{e}}} E I(x) \mathbf{C}^{-\mathbf{1} \mathbf{T}} \frac{\partial \boldsymbol{\Phi}_{\psi}}{\partial x} \frac{\partial \boldsymbol{\Phi}_{\psi}}{\partial x} \mathbf{C}^{-\mathbf{1}} \mathrm{d} x \\
& \mathbf{k}_{\mathbf{s}}=\int_{0}^{L_{\mathrm{e}}} \kappa G S(x) \mathbf{C}^{-\mathbf{1} \mathbf{T}} \boldsymbol{\Phi}_{\mathbf{s}}^{\mathbf{T}} \boldsymbol{\Phi}_{\mathbf{s}} \mathbf{C}^{-\mathbf{1}} \mathrm{d} x
\end{aligned}
$$

Conditions limites : À l'extrémité encastrée, on impose : $w(0, t)=0$ et $\psi=0(s=-\partial w / \partial x)$. À l'extrémité libre, on impose : $s=0$

Assemblage : À partir de la topologie du maillage permettant de localiser les degrés de liberté de chaque élément dans la numérotation globale, on procède à l'assemblage des matrices élémentaires. Cet assemblage permet d'exprimer les énergies cinétique $T$ et potentielle $U$ de la poutre complète à partir de la sommation de toutes les énergies élémentaires. La procédure est classique et conduit à : $T=\frac{1}{2} \dot{\mathbf{Q}}^{\mathbf{T}} \mathbf{M}_{\mathbf{0}} \dot{\mathbf{Q}}, U=\frac{1}{2} \mathbf{Q}^{\mathbf{T}} \mathbf{K}_{\mathbf{0}} \mathbf{Q}$, où : $\mathbf{M}_{\mathbf{0}}$ et $\mathbf{K}_{\mathbf{0}}$ sont les matrices globales, respectivement de masse et de rigidité, $\mathbf{Q}$ est le vecteur des degrés de liberté de tous les nouds. Appliquant les équations de Lagrange, on obtient l'équation des vibrations libres de la poutre :

$$
\mathbf{M}_{0} \ddot{\mathbf{Q}}+\mathbf{K}_{0} \mathbf{Q}=\mathbf{0}
$$


Si $\mathbf{Q}=\zeta$ est harmonique en temps, avec la pulsation $\omega: \zeta=\bar{\zeta} e^{i \omega t}$; l'équation des vibrations libres conduit au classique problème de valeurs propres :

$$
\left(\mathbf{K}_{0}-\omega^{2} \mathbf{M}_{0}\right) \bar{\zeta}=\mathbf{0}
$$

dont les solutions non-triviales sont les modes propres associés aux pulsations propres et correspondants.

\section{Formulation de la réponse de la poutre par superposition modale}

À partir des méthodes classiques de l'analyse modale, (rappelées par exemple par Seon et al. [15]), on calcule la réponse forcée de la poutre dans la base des modes propres, lesquels sont obtenus en résolvant l'équation aux valeurs propres (10). Le système des $N$ équations découplées s'écrit :

$$
\ddot{q}_{i}+\omega_{i}^{2} q_{i}=\frac{1}{\mu_{i}} \phi_{i}\left(x_{\mathrm{c}}\right) f\left(x_{\mathrm{c}}\right), \quad i=1, \ldots, N
$$

où : $\mu_{i}$ est la masse modale et $q_{i}$ la coordonnée généralisée telle que :

$$
\mathbf{W}(x, t)=\sum_{i=1}^{N}\left[\phi_{i}(x) \Psi_{i}(x) S_{i}(x)\right]^{T} q_{i}(t)
$$

Le calcul des $q_{i}$ peut être effectué de manière classique par la méthode de Newmark. Pour cela, les équations (11) sont écrites sous la forme matricielle suivante :

$$
\begin{aligned}
& \mathbf{M}^{*}(\mathbf{t}) \ddot{\mathbf{q}}+\mathbf{C}^{*}(\mathbf{t}) \dot{\mathbf{q}}+\mathbf{K}^{*}(\mathbf{t}) \mathbf{q}=\mathbf{F}_{0}^{*}(\mathbf{t}) \\
& \mathbf{M}^{*}=\operatorname{diag}\left[\mu_{i}\right]+m \operatorname{diag}\left[\phi_{i}\left(x_{\mathrm{c}}\right)\right] \boldsymbol{\Phi}\left(x_{\mathrm{c}}\right) \\
& \mathbf{C}^{*}=2 m V \operatorname{diag}\left[\phi_{i}\left(x_{\mathrm{c}}\right)\right] \boldsymbol{\Phi}^{\prime}\left(x_{\mathrm{c}}\right) \\
& \mathbf{K}^{*}=\operatorname{diag}\left[\mu_{i} \omega_{i}^{2}\right]+m V^{2} \operatorname{diag}\left[\phi_{i}\left(x_{\mathrm{c}}\right)\right] \boldsymbol{\Phi}^{\prime \prime}\left(x_{\mathrm{c}}\right) \\
& \mathbf{F}_{\mathbf{0}}^{*}=m g\left\{\phi_{1}\left(x_{\mathrm{c}}\right) \ldots \phi_{N}\left(x_{\mathrm{c}}\right)\right\}^{T} \\
& \boldsymbol{\Phi}\left(x_{\mathrm{c}}\right)=\left[\begin{array}{ccc}
\phi 1\left(x_{\mathrm{c}}\right) & \cdots & \phi_{N}\left(x_{\mathrm{c}}\right) \\
\vdots & \cdots & \vdots \\
\phi 1\left(x_{\mathrm{c}}\right) & \cdots & \phi_{N}\left(x_{\mathrm{c}}\right)
\end{array}\right] \\
& \boldsymbol{\Phi}^{\prime}=\partial \boldsymbol{\Phi} / \partial x \quad \boldsymbol{\Phi}^{\prime \prime}=\partial^{2} \boldsymbol{\Phi} / \partial x^{2}
\end{aligned}
$$

avec les conditions initiales nulles.

Cependant, pour éviter d'avoir à inverser des matrices d'itération à chaque pas de temps, nous proposons de calculer au préalable la force de contact $f$, puis d'en déduire les $q_{i}$ explicitement. Compte tenu du développement modal du déplacement $w$, on peut écrire :

$$
\begin{aligned}
& f=f_{0}-m \sum_{i=1}^{N} \phi_{i}\left(x_{\mathrm{c}}\right) \ddot{q}_{i}+2 V \phi_{i}{ }^{\prime}\left(x_{\mathrm{c}}\right) \dot{q}_{i}-\dot{V} \phi_{i}{ }^{\prime}\left(x_{\mathrm{c}}\right) q_{i} \\
& -V^{2} \phi_{i}{ }^{\prime \prime}\left(x_{\mathrm{c}}\right) q_{i} \\
& f_{0}=m\left[g-\dot{V} w_{0}{ }^{\prime}\left(x_{\mathrm{c}}\right)-V^{2} w_{0}{ }^{\prime \prime}\left(x_{\mathrm{c}}\right)\right]
\end{aligned}
$$

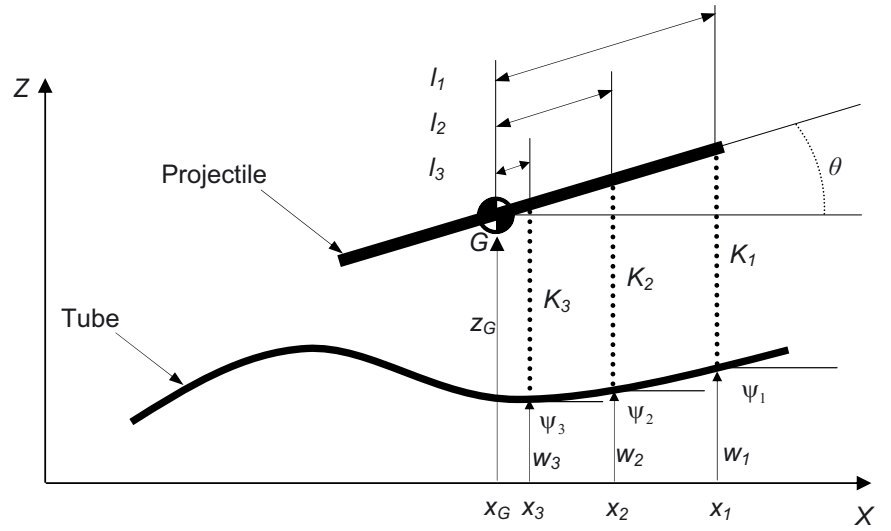

Fig. 1. Schématisation du projectile.

Si on effectue l'approximation des dérivées temporelles par des différences finies centrées, on a, pour tout $i, 1 \leq$ $i \leq N$, avec : $q_{i \mathrm{~s}}=q_{i}(t+\Delta t), q_{i \mathrm{p}}=q_{i}(t-\Delta t)$ :

$$
\begin{aligned}
\ddot{q}_{i}= & \frac{q_{i \mathrm{~s}}-2 q_{i}+q_{i \mathrm{p}}}{\Delta t^{2}}=\frac{1}{\mu_{i}} \phi_{i}\left(x_{\mathrm{c}}\right) f\left(x_{\mathrm{c}}\right)-\omega_{i}^{2} q_{i} \\
\dot{q}_{i}= & \frac{q_{\text {is }}-q_{i \mathrm{p}}}{2 \Delta t}=\frac{q_{i}-q_{i \mathrm{p}}}{\Delta t}+\frac{\ddot{q}_{i}}{2} \Delta t=\frac{q_{i}-q_{i \mathrm{p}}}{\Delta t} \\
& +\frac{\Delta t}{2}\left[\frac{\phi_{i}\left(x_{\mathrm{c}}\right)}{\mu_{i}} f\left(x_{\mathrm{c}}\right)-\omega_{i}^{2} q_{i}\right] \\
f= & f_{0}-m f\left(x_{\mathrm{c}}\right) \sum_{i=1}^{N}\left\{\phi_{i}^{2}\left(x_{\mathrm{c}}\right) / \mu_{i}+V \phi_{i}{ }^{\prime}\left(x_{\mathrm{c}}\right) \phi_{i}\left(x_{\mathrm{c}}\right) / \mu_{i}\right\} \\
& -\sum_{i=1}^{N}\left\{-\phi_{i}\left(x_{\mathrm{c}}\right) \omega_{i}^{2} q_{i}+2 V \phi_{i}{ }^{\prime}\left(x_{\mathrm{c}}\right) q_{i}-q_{i \mathrm{p}} / \Delta t\right. \\
& \left.+V \Delta t \phi_{i}{ }^{\prime}\left(x_{\mathrm{c}}\right) \omega_{i}^{2} q_{i}+\dot{V} \phi_{i}{ }^{\prime}\left(x_{\mathrm{c}}\right) q_{i}+V^{2} \phi_{i}{ }^{\prime \prime}\left(x_{\mathrm{c}}\right) q_{i}\right\}
\end{aligned}
$$

soit :

$$
f=f_{0}+C_{0} f+C_{1} \Rightarrow f=\frac{f_{0}}{1-C_{0}}+\frac{C_{1}}{1-C_{0}}
$$

Connaissant $f$, on peut calculer les coordonnées

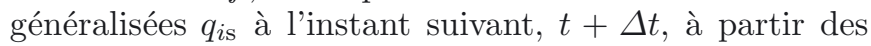
valeurs de $q_{i}$ à l'instant $t$ et de $q_{i p}$ à l'instant précédent :

$$
q_{i \mathrm{~s}}=2 q_{i}-q_{i \mathrm{p}}+\Delta t^{2} \frac{\phi_{i}\left(x_{\mathrm{c}}\right)}{\mu_{i}} f-\omega_{i}^{2} q_{i} \quad i=1, \ldots N .
$$

\section{Calcul explicite de la réponse de la poutre à un oscillateur en mouvement}

Le projectile est représenté par un barreau rigide. Il possède trois contacts (appelés «portées ») avec le tube (Fig. 1), situés à des distances respectives $l_{1}, l_{2}, l_{3} \mathrm{du}$ centre de gravité. Ces contacts sont schématisés par des ressorts de raideurs respectives $K_{1}, K_{2}, K_{3}$. De plus on 
suppose qu'il existe une raideur $C$ s'opposant à la rotation autour de l'axe $Y$. Avec les hypothèses de Timoshenko, les équations du mouvement de la poutre à section et inertie variables s'écrivent avec $w$ et $\psi$ :

$$
\begin{aligned}
& \rho S \frac{\partial^{2} w}{\partial t^{2}}=\kappa G \frac{\mathrm{d} S}{\mathrm{~d} x}\left(\frac{\partial w}{\partial x}-\psi\right)+\kappa S G\left(\frac{\partial^{2} w}{\partial x^{2}}-\frac{\partial \psi}{\partial x}\right)+P \\
& \rho I \frac{\partial^{2} \psi}{\partial t^{2}}=\kappa S G\left(\frac{\partial w}{\partial x}-\psi\right)+E \frac{\mathrm{d} I}{\mathrm{~d} x} \frac{\partial \psi}{\partial x}+E I \frac{\partial^{2} \psi}{\partial x^{2}}+Q
\end{aligned}
$$

où $\kappa$ est le coefficient correcteur de cisaillement donné par Cowper [16] tel que : $\kappa=\left[6(1+\nu)\left(1+r^{2}\right)^{2}\right] /[(7+$ $\left.6 \nu)\left(1+r^{2}\right)^{2}+(20+12 \nu) r^{2}\right],(r$ est le rapport entre le rayon intérieur et le rayon extérieur).

Efforts de contact. Le projectile est soumis à deux types d'efforts qui sont les forces élastiques et le moment de rappel. La force élastique s'exprime par : $F_{x}=$ $-K_{x}\left(z_{x}-w_{x}\right)$, avec : $x \equiv 1,2$, ou 3. $z_{x}=z_{\mathrm{G}}+l_{x} \sin \theta(t)$ désigne le déplacement suivant $Z,(\sin \theta \approx \theta)$, et $w$ le déplacement du tube :

$$
\begin{aligned}
F_{1} & =K_{1}\left[z_{G_{\mathrm{p}}}(t)+l_{1} \theta(t)-w_{1}(t)\right], \text { si } x=x_{1} \\
P\left(x_{1}\right) & =\frac{F_{1}}{\Delta x} \delta\left(x-x_{1}\right) \\
F_{2} & =K_{2}\left[z_{G_{\mathrm{p}}}(t)+l_{2} \theta(t)-w_{2}(t)\right], \text { si } x=x_{2} \\
P\left(x_{2}\right) & =\frac{F_{2}}{\Delta x} \delta\left(x-x_{2}\right) \\
F_{3} & =K_{3}\left[z_{G_{\mathrm{pl}}}(t)+l_{3} \theta(t)-w_{3}(t)\right], \text { si } x=x_{3} \\
P\left(x_{3}\right) & =\frac{F_{3}}{\Delta x} \delta\left(x-x_{3}\right)
\end{aligned}
$$

Le moment de rappel au niveau de la ceinture est proportionnel à : $\theta-\psi\left(x_{2}\right)$.

$$
\begin{aligned}
M_{2}(x, t) & =-C\left[\theta(t)-\psi\left(x_{2}, t\right)\right], \text { si } x=x_{2} \\
Q\left(x_{2}\right) & =\frac{M_{2}}{\Delta x} \delta\left(x-x_{2}\right)
\end{aligned}
$$

Appliquons le principe fondamental de la dynamique au centre de gravité du projectile afin d'obtenir l'interaction entre le projectile et la poutre :

$$
\begin{aligned}
m \ddot{x}_{G_{\mathrm{p}}}(t)= & F_{\mathrm{p}}(t) \\
m \ddot{z}_{G_{\mathrm{p}}}(t)= & F_{1}(t)+F_{2}(t)+F_{3}(t) \\
I_{\mathrm{p}} \ddot{\theta}(t)= & -F_{2}(t) l_{2}+F_{1}(t) l_{1}+F_{3}(t) l_{3} \\
& +F_{\mathrm{p}}(t) d+M(t)
\end{aligned}
$$

avec: $F_{\mathrm{p}}(t)=P_{\mathrm{c}}(t) S_{\mathrm{p}}$, où $P_{\mathrm{c}}$ est la pression à l'arrière de la munition, $S_{\mathrm{p}}$ la surface projetée de la face arrière du projectile, et $d$ le décalage du centre de gravité sur $Z$ au départ du tir. La pression $P_{\mathrm{c}}(t)$ est prise, par hypothèse, uniforme dans toute la partie située en arrière du projectile à chaque instant $t . P_{\mathrm{c}}(t)$ est déduite de mesures expérimentales. Les équations couplées sont discrétisées par des différences finies centrées. La réponse de la poutre et le mouvement du barreau à l'instant « suivant $» t+\Delta t$, sont exprimés explicitement en fonction des déplacements à l'instant « actuel » $t$, et à l'instant « précédent $» t-\Delta t$. Le schéma explicite tient compte des conditions limites. En $x=0$, la poutre est encastrée, d'où : $w=0$, et $\psi=0$. En $x=L$, l'extrémité de la poutre est libre, l'effort tranchant et le moment de flexion sont nuls : $\partial w / \partial x-\psi=0$, $\partial \psi / \partial x=0$.

\section{Résultats et discussion}

Dans un premier temps, nous analysons les résultats des modèles dits « simplifiés », permettant de connaître la réponse de la poutre parcourue par une masse (Sect. 3) et la réponse de la poutre à l'oscillateur en mouvement (Sect. 4). Dans un second temps, ces modèles simplifiés, sont comparés aux résultats de modèles développés à l'aide de codes numériques plus élaborés : le code SIMBAD et le code LS-Dyna.

\subsection{Analyse des modèles simplifiés}

Les calculs ont été effectués avec les données suivantes : $E=2,1 \times 10^{11} \mathrm{~Pa}, \rho=7800 \mathrm{~kg} \cdot \mathrm{m}^{-3}, \nu=0,3, L=$ $3 \mathrm{~m}, d_{\text {ext }}=(90-13,33 x) \times 10^{-3} \mathrm{~m}, d_{\text {int }}=40 \times 10^{-3} \mathrm{~m}$, masse en mouvement $m=0,5 \mathrm{~kg}$. Pour simplifier l'interprétation, une loi de vitesse affine (durée totale de parcours de $3 \mathrm{~ms}$ ) a été choisie :

$$
V(t)=3,333 \times 10^{5} t+500
$$

et la déformée statique : $w_{0}(x)=10^{-3}(1-\cos \pi x / 2 L)$ (en mètre) a été appliquée (elle est voisine de celle due au poids propre de la poutre).

\subsubsection{Masse en mouvement}

Les simulations effectuées ont montré que la superposition modale ${ }^{1}$ était la mieux adaptée au calcul de la force de contact (Éq. (16)) caractérisant l'interaction entre la masse et la poutre. D'autres méthodes peuvent être envisagées pour le calcul de cette force, par exemple à partir d'une discrétisation par des éléments-finis [17] ou par des différences finies. Mais le calcul est alors très influencé par les perturbations numériques lors de l'évaluation des dérivées figurant dans l'expression de $f$ (en particulier : $\left.\ddot{w}+2 V \dot{w}^{\prime}+V^{2} w^{\prime \prime}+\dot{V} w^{\prime}\right)$.

L'évolution de cette force le long du parcours est représentée dans la figure 2 . Un tracé plus restreint (Fig. 3) montre son évolution particulièrement rapide vers l'extrémité libre et les intensités importantes qu'elle atteint. La courbe (a) correspond à $\kappa=0,59$, la courbe (b)

\footnotetext{
1 La superposition modale est effectuée avec 150 modes et une résolution spatiale $L / 700$.
} 


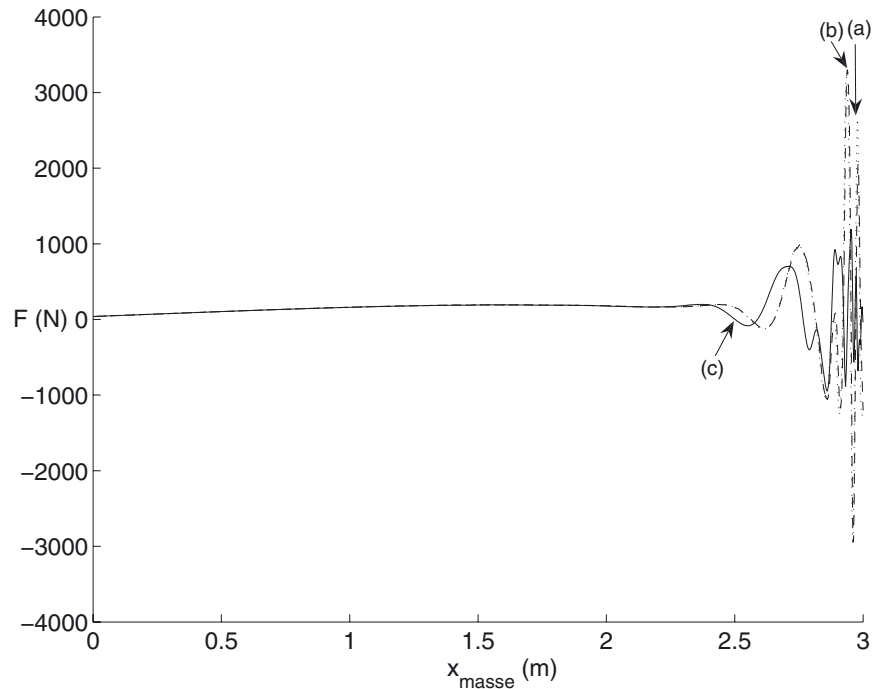

Fig. 2. Force lors du parcours du projectile.

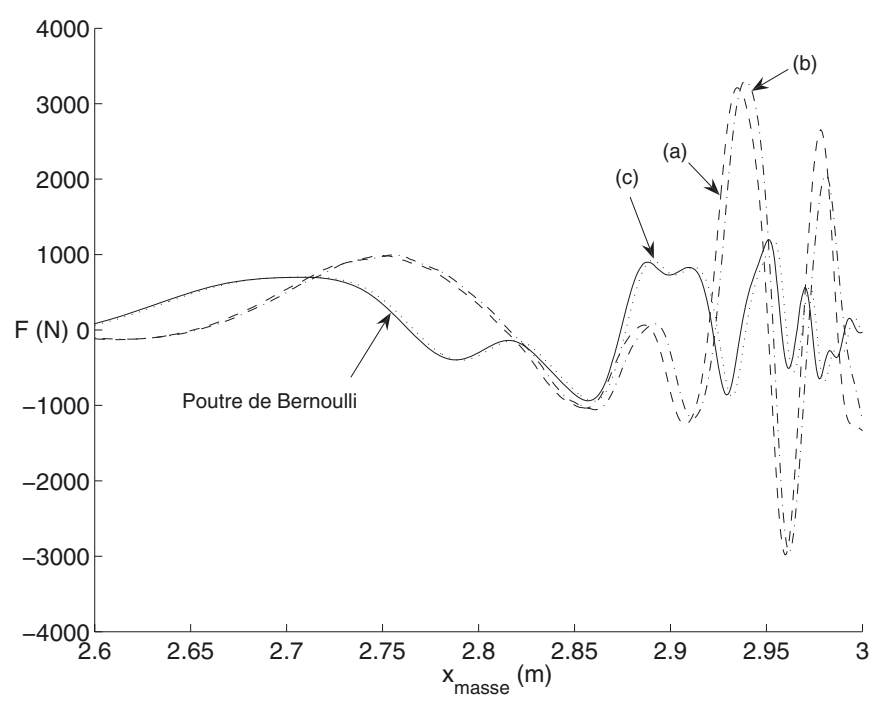

Fig. 3. Force lors du parcours du projectile au voisinage de l'extrémité du tube.

correspond au coefficient donné par la formule de Cowper [16]. Lorsque le coefficient correcteur de cisaillement $\kappa$ devient très grand, le comportement dynamique de la poutre de Timoshenko tend vers celui d'une poutre d'Euler-Bernoulli; on peut s'en convaincre d'après la figure 3 où la poutre de Bernoulli (traitée ici avec son inertie de rotation ${ }^{2}$ ) et la poutre de Timoshenko ont des réponses très voisines si $\kappa=10^{4}$ (c). Il est très clair, par contre, que les hypothèses de Timoshenko (prise en compte du cisaillement et de l'inertie de rotation) et d'Euler-Bernoulli conduisent à des réponses très différentes lorsque la masse en mouvement se rapproche de l'extrémité libre. C'est ce qui est illustré par

\footnotetext{
${ }^{2}$ Les modes et fréquences propres en sont calculés d'après Martinez-Castro et al. [18].
}

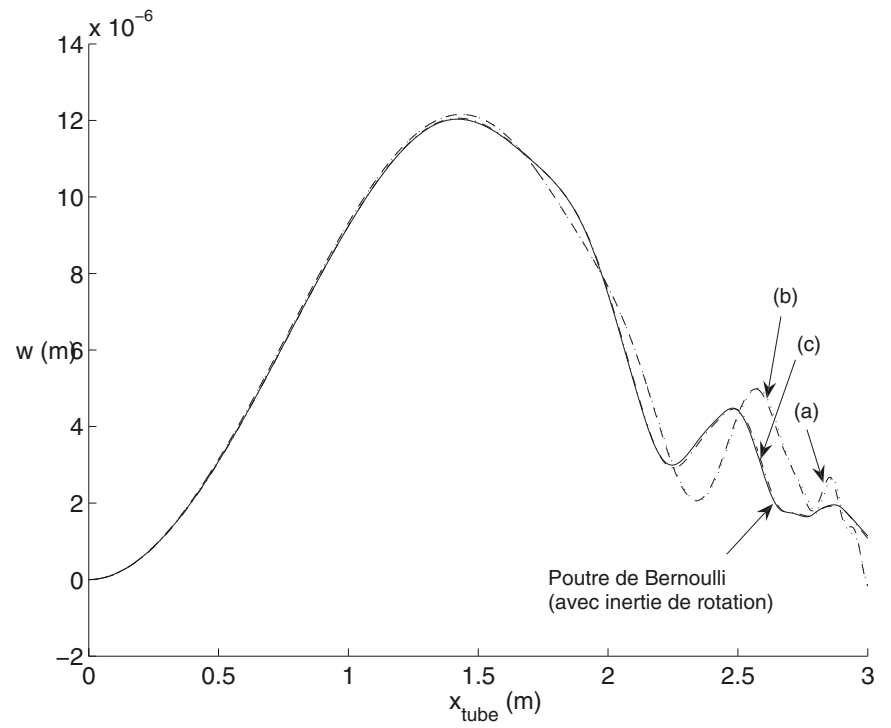

Fig. 4. Déformée du tube à l'instant de la sortie du projectile (Bernoulli et trois valeurs de $\kappa$ ).

les figures 2 et 3 pour la force de contact, mais aussi, par la figure 4 , pour le déplacement $w$. On vérifie ainsi que les hypothèses de Timoshenko sont adaptées pour décrire les vibrations de faibles longueurs d'ondes et de hautes fréquences.

\subsubsection{Oscillateur en mouvement à accélération constante}

Les raideurs considérées sont les suivantes : $K_{1}=$ $2,325 \times 10^{7} \mathrm{~N} \cdot \mathrm{m}^{-1}, K_{2}=4,466 \times 10^{6} \mathrm{~N} \cdot \mathrm{m}^{-1}, K_{3}=$ $8 \times 10^{7} \mathrm{~N} . \mathrm{m}^{-1}, C=1.207 \times 10^{3} \mathrm{~N} .{\mathrm{m} . \mathrm{rad}^{-1}}^{-1}$ La figure 5 représente les déformées finales $w$ (lorsque l'oscillateur atteint l'extrémité libre, c'est-à-dire la « bouche »du tube) et la figure 6 représente les rotations $\psi$ finales des sections droites du tube.

Ces figures sont destinées à illustrer l'influence de la variabilité de la loi de vitesse $V(t)$ du projectile dans le tube. En effet, la vitesse et l'accélération peuvent varier (légèrement) d'un tir à l'autre. Les vitesses retenues en $x=L$ varient entre $1450 \mathrm{~m} . \mathrm{s}^{-1}$ et $1550 \mathrm{~m} . \mathrm{s}^{-1}$; la vitesse d'entrée dans le tube est de $500 \mathrm{~m} . \mathrm{s}^{-1}$. Il est intéressant de faire cette étude, car une partie des conditions initiales $\mathrm{du}$ vol du projectile est contenue dans ces courbes. On peut remarquer que la vitesse a peu d'influence sur l'allure générale des courbes lorsque l'on considère les deux premiers tiers du tube (où $w$ et $\psi$ ont des longueurs d'onde assez grandes). C'est au-delà du dernier tiers du tube que l'influence de la variation de l'accélération se manifeste.

D'autres informations sont nécessaires pour compléter les conditions initiales du vol comme la vitesse angulaire $\dot{\theta}$ et latérale $\dot{z}$ du projectile à la sortie du tube. C'est ce que l'on peut observer sur les figures 7 et 8 en fonction de la vitesse à la bouche du tube. Ces figures montrent que les vitesses angulaire $\dot{\theta}$ ou de translation $\dot{z}$ augmentent, en 

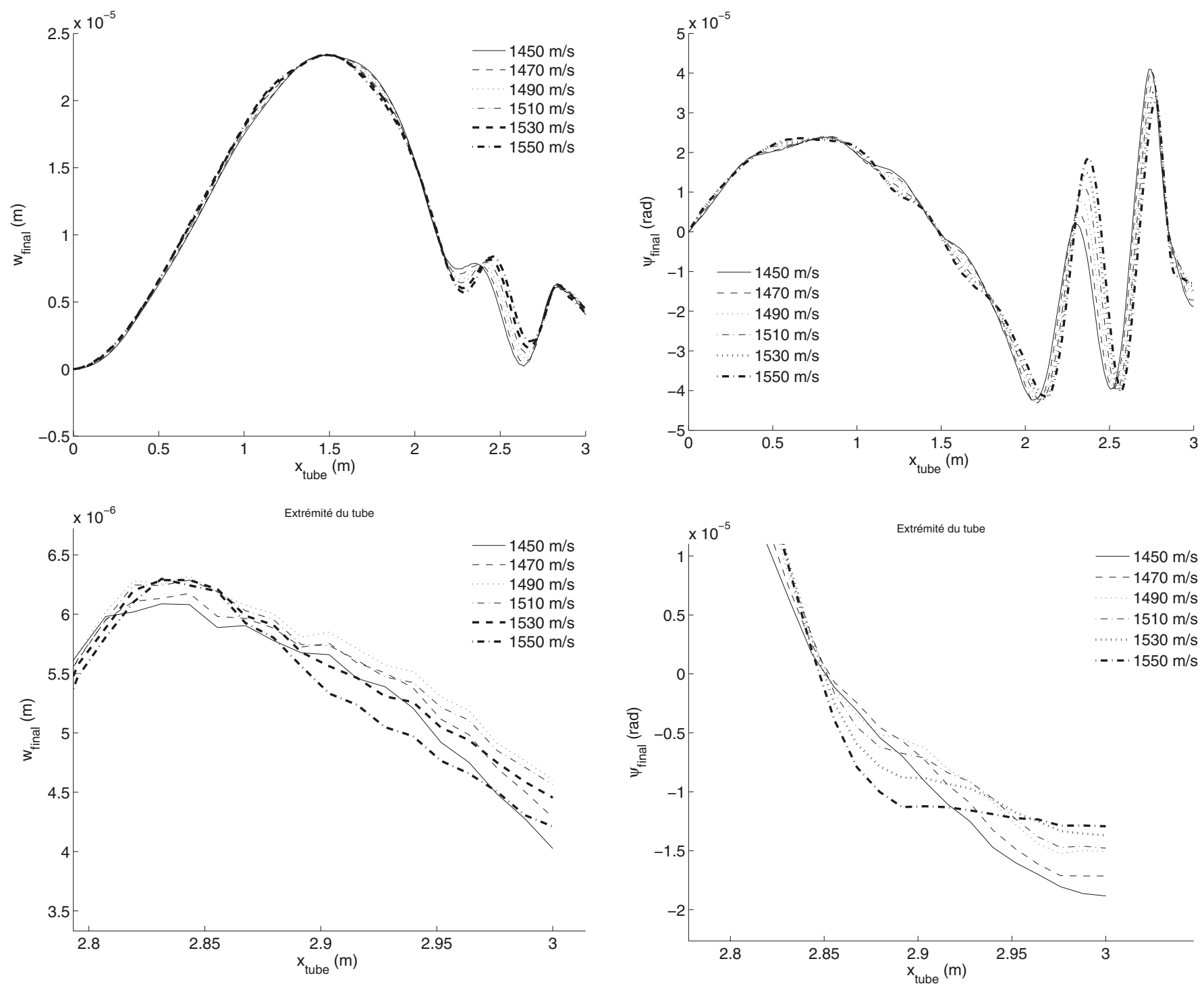

Fig. 5. Déformée du tube à l'instant de la sortie du projectile pour une plage de vitesses.

valeur absolue, avec l'augmentation de la vitesse du projectile à la bouche. En effet, les mouvements de l'extrémité libre du tube vont se produire à des fréquences d'autant plus grandes que l'accélération $\dot{V}(t)$ est élevée; cela se traduit par des vitesses en translation $\dot{w}$ et en rotation $\dot{\psi}$ d'autant plus grandes, et, par couplage, par des vitesses $\dot{z}$ et $\dot{\theta}$ d'autant plus élevées.

\subsubsection{Oscillateur en mouvement avec une accélération dépendante du temps}

En pratique, la déformée initiale du tube est la somme de la déformée due à son poids propre et des imperfections géométriques (notées $\tilde{w}_{0}(x)$ ). Le profil $\tilde{w}_{0}(x)$ du tube est déduit de mesures optiques. La figure 9 montre le profil $\tilde{w}_{0}(x)$ retenu pour le calcul de la réponse du tube (la déformée due au poids propre n'est pas incluse ici).

Fig. 6. Rotation des sections du tube à l'instant de la sortie $\mathrm{du}$ projectile pour une plage de vitesses.

On applique maintenant à l'oscillateur une accélération résultant de la pression des gaz $P_{\mathrm{c}}(t)$ régnant dans l'espace en arrière du projectile (celle-ci a été déterminée expérimentalement) en appliquant l'équation (24). La vitesse de l'oscillateur est nulle ${ }^{3}$ en $x=0$ et vaut $1480 \mathrm{~m} . \mathrm{s}^{-1}$ en $x=L$. Les raideurs et moments de rappels sont les mêmes que précédemment. Sur la figure 10 on a représenté la déformée dynamique du tube (déplacement $w$ ) pendant le temps de parcours de l'oscillateur représenté par un point. On y voit que le déplacement du projectile est influencé par des ondes réfléchies depuis l'extrémité libre dès le tiers du temps total de parcours (environ $1 \mathrm{~ms}$ ). En effet, le mouvement de l'extrémité libre débute à partir de $1 \mathrm{~ms}$ (Fig. 11).

\footnotetext{
${ }^{3}$ La vitesse nulle correspond à une pression nulle au départ
} du tube. 


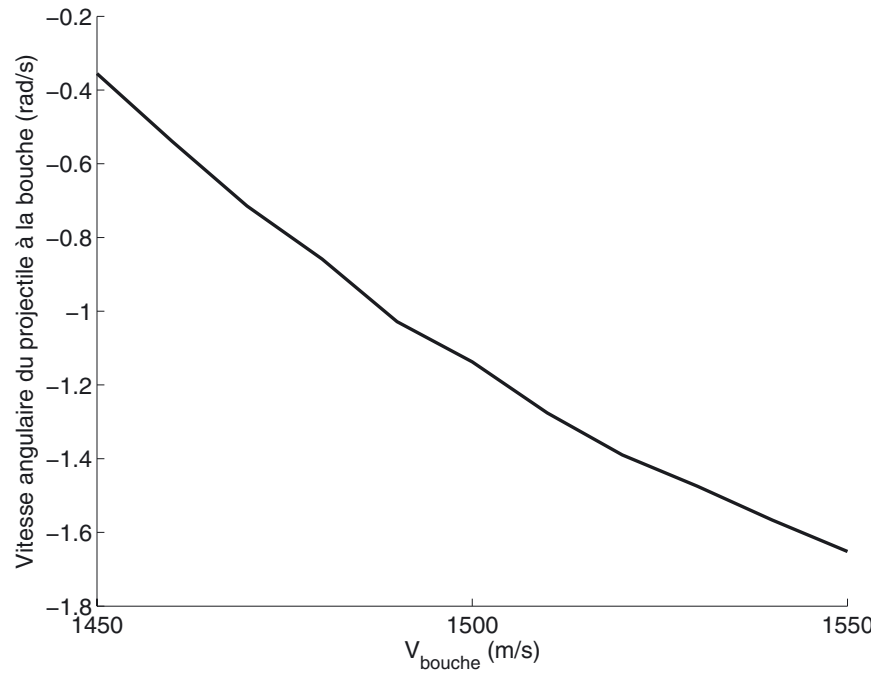

Fig. 7. Vitesse angulaire du projectile en fonction de sa vitesse à la bouche.

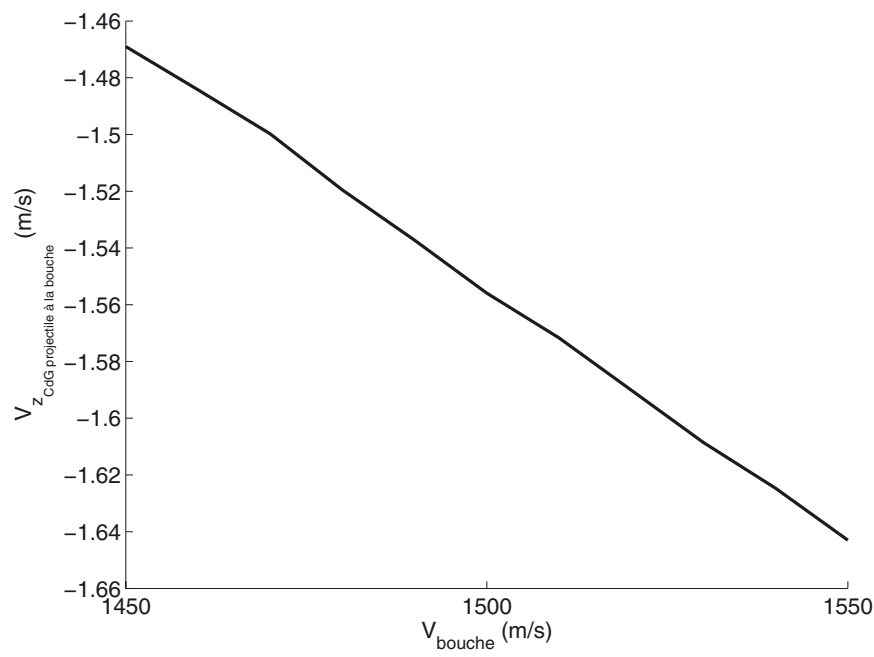

Fig. 8. Vitesse transverse du projectile en fonction de sa vitesse à la bouche.

\subsubsection{Convergence des réponses à une masse mobile et à un oscillateur en mouvement}

Pour résumer les résultats issus de cette première modélisation, le graphique de la figure 12 permet une comparaison des déformées de la poutre tronconique parcourue par une masse pour la courbe (a) et par un oscillateur pour la courbe (b) $(m=0,5 \mathrm{~kg})$. Ici, l'oscillateur n'est relié à la poutre que par un seul ressort, de raideur $10^{8} \mathrm{~N} . \mathrm{m}^{-1}$. On vérifie que lorsque la raideur est très grande, les deux systèmes ont des réponses convergentes (comme l'avait montré Pesterev et al. [12] avec des chargements à vitesses constantes et des poutres uniformes). Cependant, il n'est pas possible de faire tendre la raideur de l'oscillateur vers l'infini, car cela impose des pas de temps $\mathrm{d} t$ et d'espace $\mathrm{d} x$ extrêmement faibles (de l'ordre de $10^{-9} \mathrm{~s}$ et de $1 \mathrm{~mm}$ dans le cas présent) pour assurer la stabilité du schéma numérique explicite. L'application de

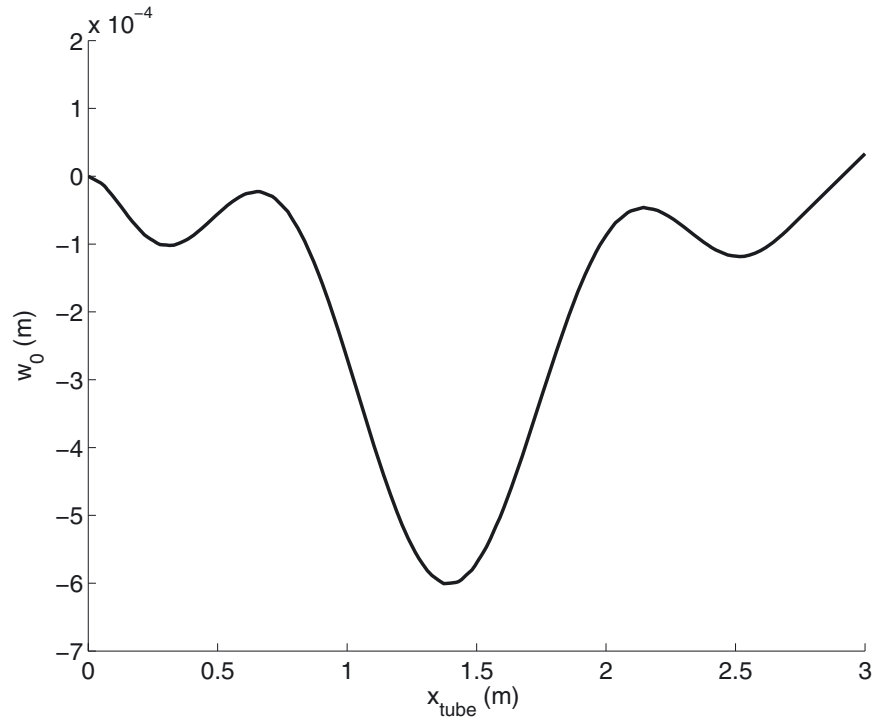

Fig. 9. Déformée initiale du tube.

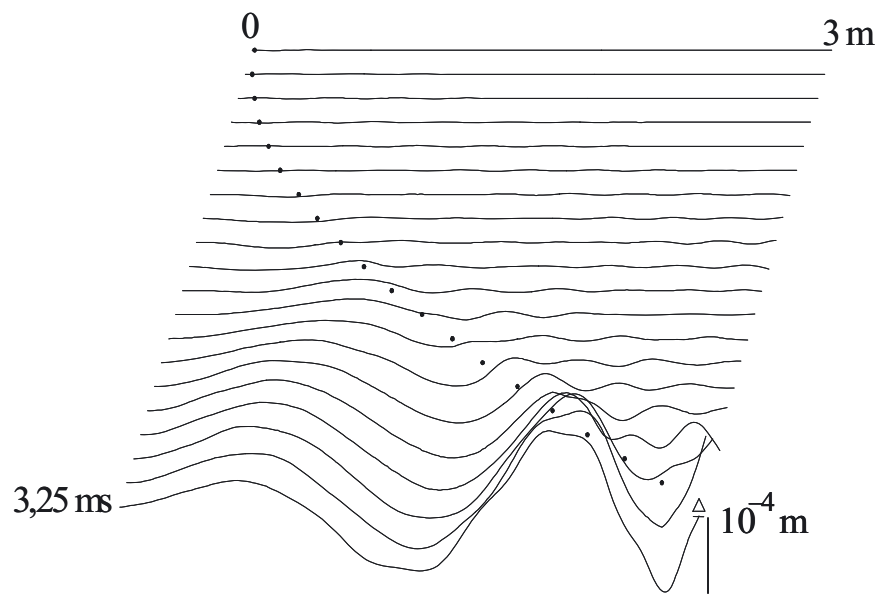

Fig. 10. Déformée du tube au cours du temps.

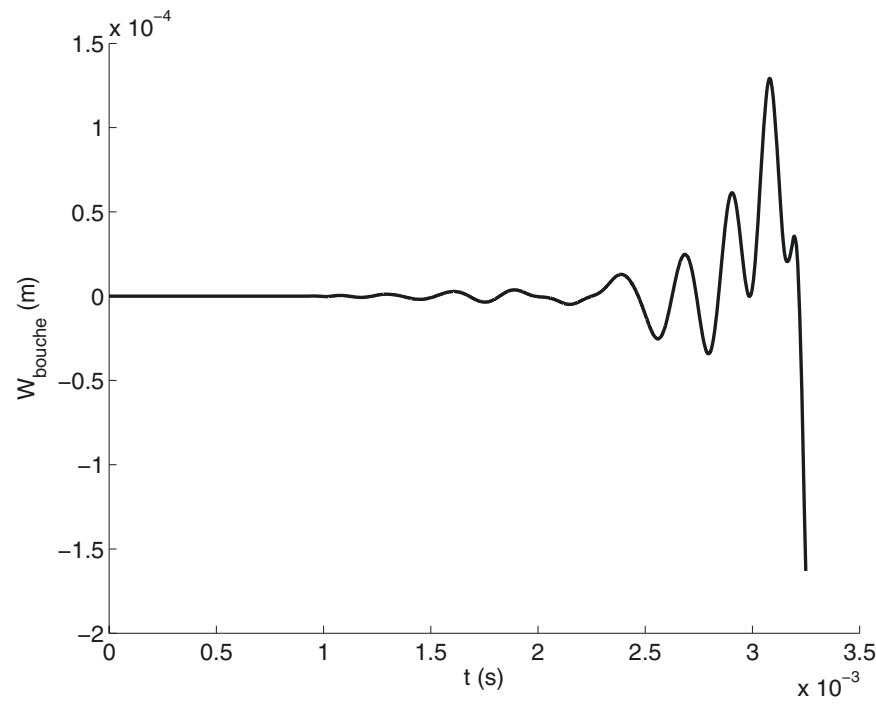

Fig. 11. Déplacement de la bouche du tube au cours du temps. 


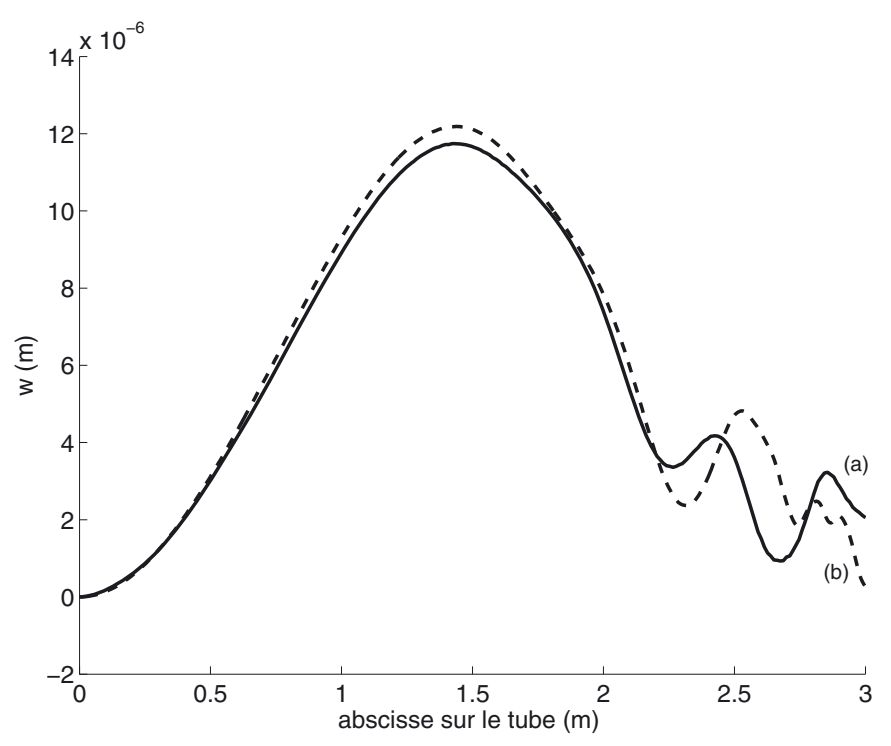

Fig. 12. Déformées du tube selon deux méthodes.

la méthode de superposition modale est alors préférable (les temps de calcul sont environ 100 fois plus courts).

\subsection{Comparaison des modèles simplifiés avec des codes industriels (SIMBAD et LS-Dyna)}

Afin de conforter la validité du modèle simplifié (oscillateur avec plusieurs ressorts), des comparaisons avec deux codes existants ont été réalisées. Le premier, SIMBAD, le moins complexe des deux, est dédié à l'univers de l'armement. Il est basé uniquement sur la théorie des poutres associée à la méthode éléments-finis. Le second, LS-Dyna, basé sur la méthode éléments-finis, est un code général tridimensionnel pour la dynamique rapide.

Dans les deux cas, les calculs ont été effectués avec les données suivantes : $E=2,1 \times 10^{11} \mathrm{~Pa}, \nu=0,3, L=2,8 \mathrm{~m}$, $d_{\text {ext }}=(90-13,33 x) \times 10^{-3} \mathrm{~m}, d_{\text {int }}=40 \times 10^{-3} \mathrm{~m}$. Le tube est plus court que précédemment pour être plus proche des cotes réelles. La même accélération provenant de la pression $P_{\mathrm{c}}(t)$ a été appliquée.

Avec le code SIMBAD, deux profils intrinsèques de tube sont étudiés : un profil sinusoïdal, et le profil $\tilde{w}_{0}(x)$ issu de mesures optiques. Avec le code LS-Dyna, on applique uniquement le profil correspondant à la déformée du poids propre.

Les données d'entrées du code SIMBAD ont été simplifiées le plus possible afin d'être au plus proche du calcul explicite de la réponse de la poutre à un oscillateur : en réduisant à une dimension d'espace le calcul, en réduisant à une seule le nombre de portées du projectile sur le tube, en rigidifiant les ressorts de frein de tir pour annuler presque totalement l'effet de recul, en rigidifiant l'encastrement pour se rapprocher au plus d'un encastrement parfait. Cependant, il est impossible de faire dégénérer le modèle SIMBAD vers le modèle simplifié,

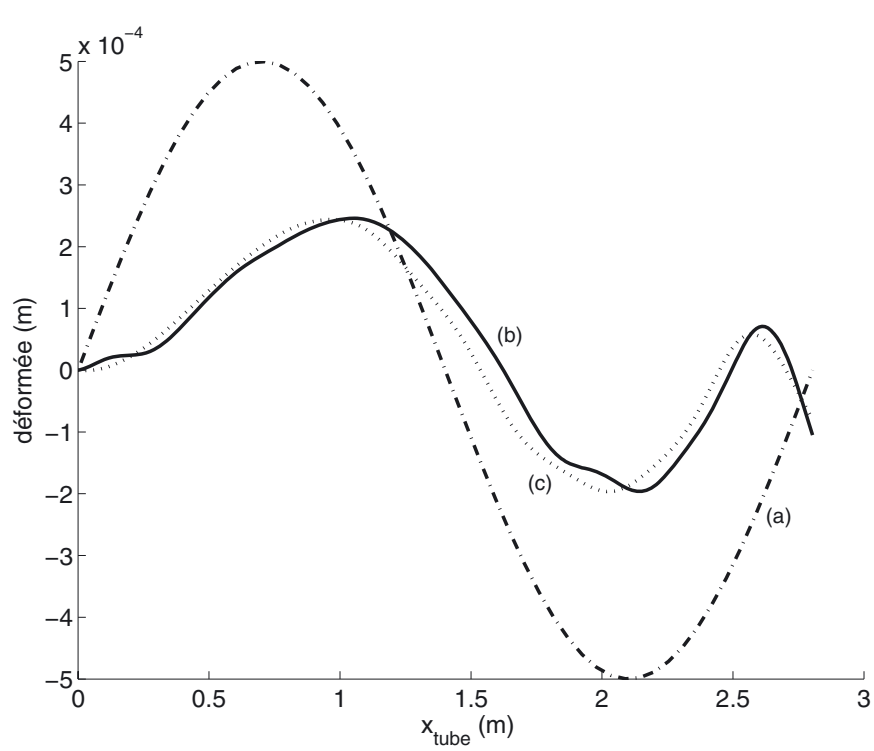

Fig. 13. Déformée du tube à l'instant de sortie du projectile pour un profil interne initial sinusoïdal.

car l'influence des paramètres pris en compte reste non négligeable, même lorsque ceux-ci ont été réduits : notamment le recul, et la raideur d'encastrement qui correspond à un guidage au niveau de la face arrière du tube. D'autre part, la raideur de l'encastrement ne peut dépasser une certaine limite $\left(10^{9} \mathrm{~N} . \mathrm{m}^{-1}\right)$, sous peine d'instabilité.

Les figures 13 et 14 présentent les déformées des tubes. La courbe (a) représente le profil initial retenu pour le calcul, la courbe (b) le résultat du code simplifié, et la courbe (c) le résultat du code SIMBAD. On peut noter que les allures et ordres de grandeurs sont semblables. Les écarts sont dus au fait qu'il n'est pas possible de simplifier suffisamment le modèle SIMBAD, notamment pour la raideur placée à l'encastrement. Cependant, ceci permet d'étayer la validité du calcul explicite simplifié de la réponse d'un tube à un oscillateur en mouvement.

Pour la comparaison avec LS-Dyna, dont on peut voir une illustration sur la figure 15, le tube tronconique est identique géométriquement à celui modélisé précédemment. Il est soumis à son poids propre $\left(\tilde{w}_{0}=0\right)$. L'accélération est déduite de la pression $P_{\mathrm{c}}(t)$ comme précédemment. Le projectile est représenté, dans ce calcul, par un cylindre de $40 \mathrm{~mm}$ de diamètre et de $40 \mathrm{~mm}$ de long. Le contact est sans frottement. Le contrôle du contact est classique. On teste la non-pénétration de facettes par d'autres facettes d'éléments. Une des surfaces est « maître » et l'autre « esclave »; c'est celle-ci qui est repoussée en cas d'interpénétration. Les éléments retenus sont des hexaèdres à un point d'intégration (éléments dits sous-intégrés) qui donnent le meilleur rapport coût efficacité lors de calculs explicites, tout en permettant d'éviter certains verrouillages numériques (en déformation) sur des éléments entièrement intégrés. Par contre, ceux-ci sont sujets aux déformations à énergie nulle (déformations en distorsion, ne modifiant pas la longueur des diagonales, 


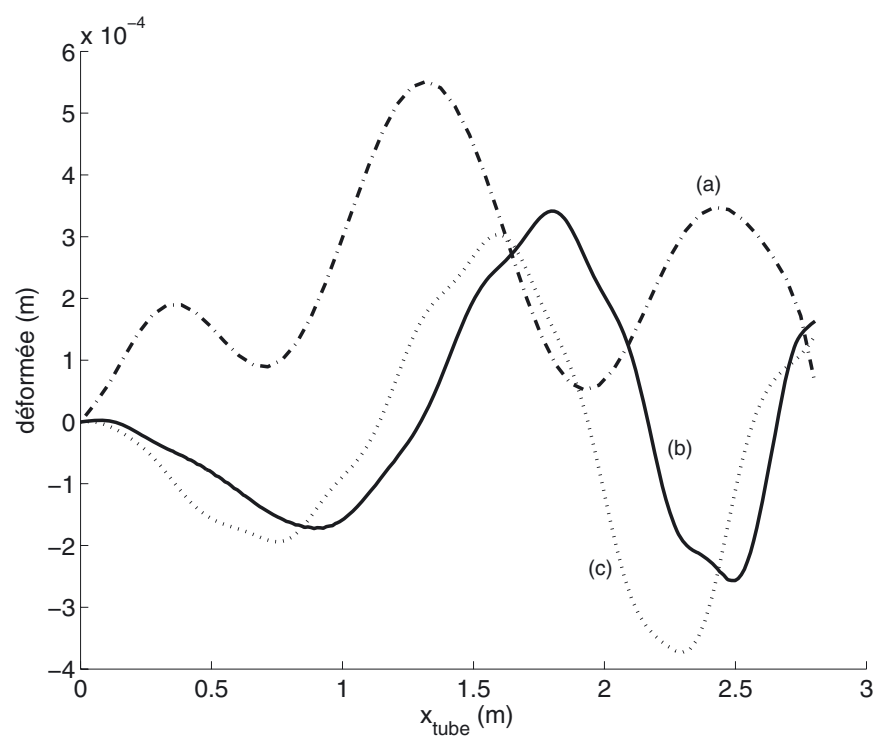

Fig. 14. Déformée du tube à l'instant de sortie du projectile pour un profil interne initial réel.

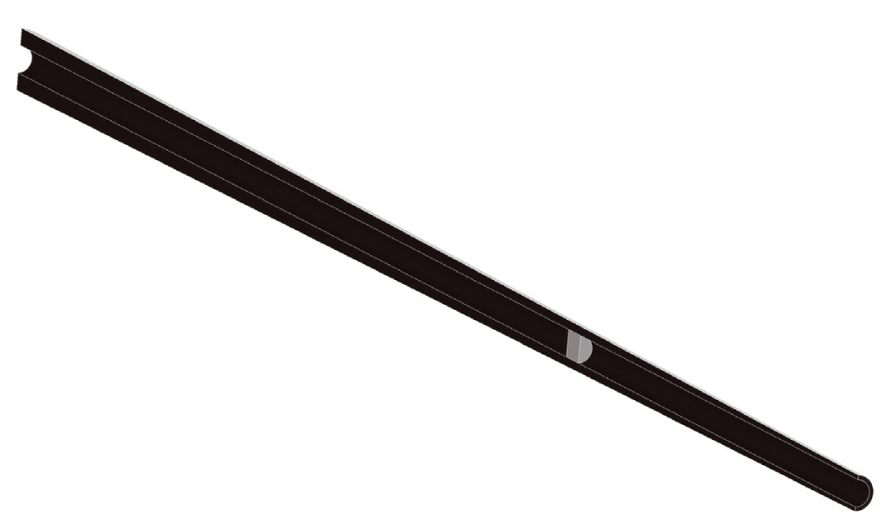

Fig. 15. Géométrie du tube pour le modèle LS-Dyna.

appelés mode d'Hourglass). On contre ces effets en rajoutant de la viscosité artificielle.

La figure 16 représente un calcul de déformations de flexion sur la peau extérieure du tube à $70 \mathrm{~cm}$ de son extrémité libre. La courbe (a) représente le résultat du modèle simplifié, et la courbe (b) le résultat du code LS-Dyna. On constate que les formes des deux signaux sont voisines. Le contenu fréquentiel apparaît voisin. Il existe par contre des différences significatives entre les amplitudes après $5 \mathrm{~ms}$. Les différences entre les résultats tiennent à la nature différente des modélisations (différences finies explicites pour le calcul simplifié, éléments-finis et algorithme explicite pour le parcours dynamique pour LS-Dyna).

\section{Conclusion}

Dans un premier temps nous avons déterminé les matrices de masse et de rigidité d'une poutre tronconique

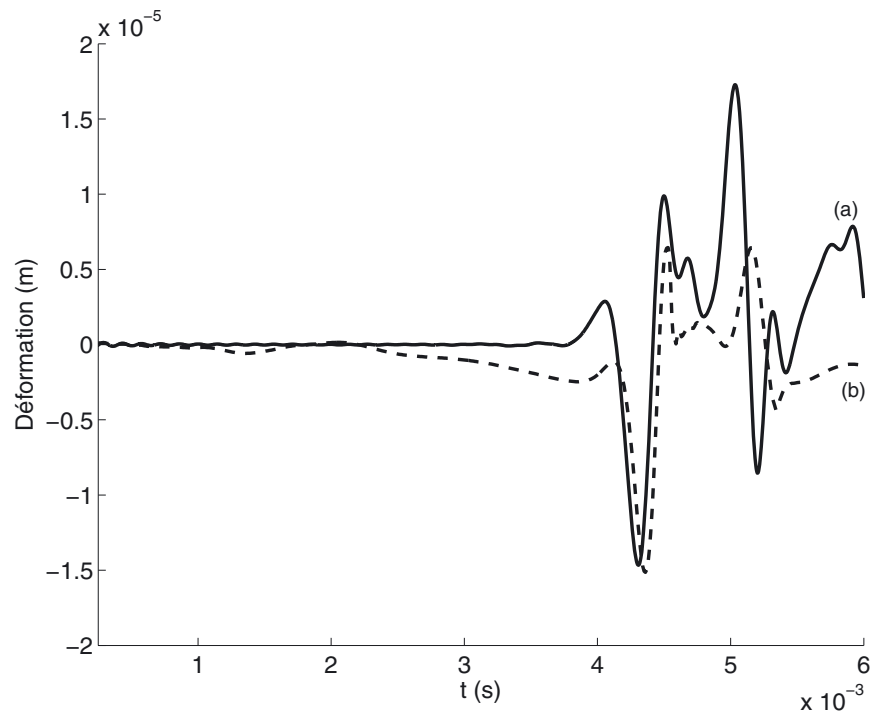

Fig. 16. Comparaison d'une déformation sur le tube.

encastrée-libre représentant un tube d'arme, pour en calculer les modes et les fréquences propres. Lorsqu'une masse en mouvement représente le chargement, l'interaction avec la dynamique de la poutre provient des termes d'inertie contenus dans l'accélération. Connaissant la base modale, deux méthodes sont possibles pour calculer la réponse de la poutre parcourue par une masse. La première, classique, consiste à projeter le chargement $f$ dans la base modale, ce qui conduit à l'équation (12). La solution peut, dans ce cas, être obtenue par l'algorithme de Newmark. La seconde, consiste à calculer d'abord la force $f$ par l'équation (16), puis à calculer les coordonnées généralisées $q_{i}$. La première méthode présente l'avantage d'être inconditionnellement stable pour le calcul des déplacements; par contre, il est alors nécessaire de calculer directement les dérivées $\ddot{w}, \dot{w}^{\prime}$ et $w^{\prime \prime}$ pour connaître $f$. Ces calculs se sont avérés extrêmement bruités numériquement et inutilisables.

Le modèle de l'oscillateur est le plus réaliste car il tient compte des raideurs de contact et permet d'introduire l'angle de rotation du barreau. Lorsque les raideurs deviennent très « grandes » le calcul explicite basé sur la méthode des différences finies nécessite des pas de temps de plus en plus petits (inférieurs à $10^{-8} \mathrm{~s}$ ) pour ne pas être instable, ce qui devient très pénalisant. Cependant, pour les raideurs extrêmes, la réponse devient comparable à celle que produit la masse en mouvement.

L'accroissement des raideurs de contact et des vitesses entraîne une diminution des longueurs d'ondes vibratoires. Pour décrire ces vibrations, le modèle de poutre de Timoshenko a été choisi. Nous avons montré que, dans certaines limites, le modèle simplifié de l'oscillateur (Sect. 4) converge avec les modèles plus complexes mis en œuvre (SIMBAD et LS-Dyna).

En conclusion, on dispose d'un outil relativement simplifié, nécessitant des temps de calculs réduits, pour étudier la dynamique en flexion de tubes 
parcourus à grandes vitesses. Les modèles présentés sont complémentaires de codes numériques plus complexes dédiés à la dynamique des structures soumises à des chargements mobiles.

Remerciements. L'équipe de ce projet tient à remercier tout particulièrement l'Europe, la Région Centre, l'université d'Orléans, le Pôle Capteur, l'A.N.R.T., sans oublier les partenaires industriels C.T.A. International et Nexter, sans lesquels le projet n'aurait pas pu voir le jour.

\section{Références}

[1] U. Lee, Separation between the flexible structure and the moving mass sliding on it, J. Sound Vibr. 209 (1998) 867-877

[2] A.V. Pesterev, B. Yang, L.A. Bergman, C.A. Tan, Response of elastic continuum carrying multiple moving oscillators, ASCE J. Eng. Mech. 127 (2001) 260-265

[3] C.R. Steele, The Timoshenko beam with a moving load, J. Appl. Mech. 35 (1968) 481-488

[4] L. Fryba, Vibration of solids and structures under moving loads, Noordhoff Publishing, Groningen, 1972

[5] J. Renard, M. Taazount, Transient responses of beams and plates subject to travelling load. Miscellaneous results, Eur. J. Mech. A/Solids 21 (2002) 301-322

[6] J.R. Rieker, M.W. Trethewey, Finite element analysis of an elastic beam structure subjected to a moving distributed mass train, Mech. Syst. Sig. Proc. 13 (1999) 31-51

[7] M.P. Cartmell, J.J. Wu, A.R. Whittaker, Dynamic responses of structures to moving bodies using combined finite element and analytical methods, Int. J. Mech. Sci. 43 (2001) 2555-2579
[8] M. Olsson, Finite element modal co-ordinate analysis of structures subjected to moving loads, J. Sound Vib. 99 (1985) $1-12$

[9] H.P. Lee, The dynamic response of a Timoshenko beam subjected to a moving mass, J. Sound Vib. 198 (1996) 249-256

[10] J.E. Akin, M. Mofid, Numerical solution for response of beams with moving mass, J. Structural Eng. 115 (1989) 120-131

[11] E.C. Ting, J. Genin, J.H. Ginsberg, A general algorithm for moving mass problem, J. Sound Vib. 33 (1974) 49-58

[12] A.V. Pesterev, L.A. Bergman, C.A. Tan, T.C. Tsao, B. Yang, On asymptotics of the solution of the moving oscillator problem, J. Sound Vib. 260 (2003) 516-536

[13] C.W.S. To, A linearly tapered beam finite element incorporating shear deformation and rotary inertia for vibration analysis, J. Sound Vibr. 78 (1981) 475-484

[14] Y.C. Hou, C.H. Tseng, A new high-order non-uniform Timoshenko beam finite element on variable twoparameter foundations for vibration analysis, J. Sound Vib. 191 (1996) 91-106

[15] M.H. Seon, B. Haym, W. Timothy, Dynamics of transversely vibrating beams using four engineering theories, J. Sound Vib. 225 (1999) 935-988

[16] G.R. Cowper, The shear coefficient in Timoshenko's beam theory, J. Appl. Mech. 33 (1966) 335-340

[17] J.R. Rieker, Y.-H. Lin, M.W. Trethewey, Discretization considerations in moving load finite element beam models, Finite elements in Analysis and Design 21 (1996) 129-144

[18] A.E. Martinez-Castro, P. Museros, A. Castillo-Linares, Semi-analytic solution in the time domain for nonuniform multi-span Bernoulli-Euler beams traversed by moving loads, J. Sound Vib. 294 (2006) 278-297 